\title{
Optimal Damping Profiles for a Heaving Buoy Wave Energy Converter
}

\author{
G.A. Nolan ${ }^{1}$, J.V. Ringwood ${ }^{1}$, W.E. Leithead ${ }^{2}$ and S. Butler ${ }^{1}$ \\ ${ }^{1}$ Department of Electronic Engineering, \\ National University of Ireland, Maynooth. \\ Maynooth, Co. Kildare, Ireland \\ ${ }^{2}$ Department of Electronics \& Electrical Engineering, \\ University of Strathclyde. \\ Strathclyde, Glasgow, UK.
}

\begin{abstract}
This paper explores optimal damping profiles for a heaving buoy wave energy converter (WEC). The approach is mathematical and the model of Eidsmoen (1995) is used as a basis. In order to permit analytical development, the model is initially simplified and an optimal damping profile is determined using numerical optimization. Having found the optimal damping profile, a semi-analytical solution methodology is developed to determine the optimal damping parameters. Finally, the procedure is validated on the original model and some aspects related to the control problem are addressed.
\end{abstract}

KEY WORDS: Wave energy; heaving buoy; latching; optimisation.

\section{INTRODUCTION}

It has been established that optimal energy recovery from point absorber wave energy devices can be achieved under a number of conditions:

1. The velocity profile of the device is in phase with the excitation force experienced by the buoy, and

2. Energy is supplied during the wave cycle to maximise the velocity excursion (Falnes, 2002).

While the second condition is rarely addressed, due to the requirement for very complex power-take-off mechanisms, a limited number of solutions to condition one have been considered. These include:

- Linear damping - the simplest case of employing a constant linear damping coefficient,

- Freewheeling (Wright et al, 2003) - the device is allowed to 'freewheel' unloaded from the extrema, allowing velocity to build up, and is then loaded after a certain velocity threshold, and

- Latching (Budal and Falnes, 1975) - the buoy is locked in position at the instant when its velocity becomes zero and then released after a fixed time period.

However, an enormous range of possibilities exist for parameterising the damping force, where all possible variations in damping force with both time (over the wave cycle) and velocity can be considered. The novel approach in this paper is to employ a parametric profile for damping (with time) where the parameters, initially assigned to give a linear damping profile, are optimised to maximise energy absorbed per wave cycle.

The approach adopted centres on simplification of the detailed hydrodynamic model of the heaving buoy as developed by Eidsmoen (1995). This is necessary, since the equations of motion for the system rapidly become intractable due to the inclusion of a damping coefficient which is dependent on one of the primary system variables. In spite of this simplification, an analytical solution for the optimal damping profile is not possible and some recourse is made to numerical techniques for the optimisation. The problem is conveniently solved, following the employment of an evolutionary algorithm, which surprisingly returns latching as the optimal damping profile.

Following this discovery, the paper then proceeds to determine the optimal latching time, in terms of the parameters of the excitation force and the system parameters. Unsurprisingly, this problem also does not permit a complete analytical solution. However, an analytical equation is developed which can be very simply and efficiently optimized to give the optimum latching time.

Finally, some consideration is given to the control problem of a point absorber WEC

\section{MATHEMATICAL MODEL}

The mathematical model described here was developed by Harvard Eidsmoen and is documented in his $\mathrm{PhD}$ thesis (Eidsmoen, 1995). The model is of a cylindrical buoy of the dimensions given in Fig. 1. The motion of the buoy is relative to a fixed reference and is constrained to heave motion only. 


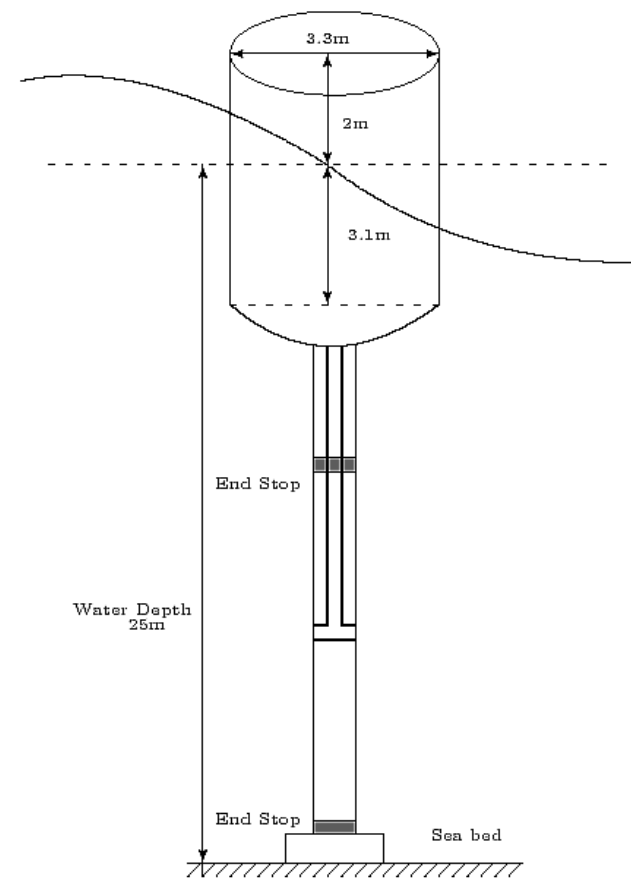

Figure 1. Geometry of the buoy

The total wave force on the buoy can be written as:

$F_{w}(t)=F_{e}(t)+F_{r}(t)$

where the excitation force, $F_{e}(t)$, is given by:

$F_{e}(t)=f(t) * \eta(t)=\int_{-\infty}^{\infty} \eta(\tau) f(t-\tau) d \tau$

and the radiation force, $F_{r}(t)$, is given (Cummins, 1962) by:

$$
\begin{aligned}
F_{r}(t) & =-z_{r}(t) * \dot{x}(t)=-m_{r}(\infty) \ddot{x}(t)-k(t) * \dot{x}(t) \\
& =-m_{r}(\infty) \ddot{x}(t)-\int_{-\infty}^{t} \dot{x}(\tau) k(t-\tau) d \tau
\end{aligned}
$$

In $F_{e}(t), \quad \eta(t)$, the elevation due to the incident wave at the origin, is convolved with, $f(t)$, the excitation force kernel. In $F_{r}(t), m_{r}(\infty)$ is the added mass of the buoy at infinite frequency, $x(t)$ is the vertical displacement of the heaving buoy from rest and $k(t)$ is the radiation force kernel. The kernels, of which $f(t)$, contrary to $k(t)$, is non-causal, can be obtained from the frequency domain expressions for the hydrodynamic parameters of the buoy and are both included in (Eidsmoen, 1995)

The complete equation of motion is given by:

$m_{b} \ddot{x}(t)+S x(t)=F_{w}(t)+F_{f}(t)+F_{u}(t)+F_{c}(t)+F_{m}$

where $m_{b}$ is the mass of the buoy, $S$ is the hydrostatic stiffness of the buoy, $F_{f}(t)$ is the friction force, $F_{u}(t)$ is the load force as described by Eq. $6, F_{c}(t)$ is the force due to the end stop device and $F_{m}$ is the net buoyancy force. Including the individual expressions for all the forces, Eq. 4 can be written as:

$$
\begin{aligned}
\ddot{x}(t)=\frac{1}{m_{b}+m_{r}(\infty)}\left\{\int_{-\infty}^{\infty} \eta(\tau) f(t-\tau) d \tau-\int_{-\infty}^{t} k(t-\tau) \dot{x}(\tau) d \tau\right. \\
\left.\quad-R_{f} \dot{x}(t)-S x(t)+F_{u}(t)+F_{c}(t)+F_{m}\right\}
\end{aligned}
$$

where $R_{f}$ is the friction resistance coefficient. $F_{u}(t)$, the load force, is the force representing the damping or hydraulic resistance due to the power take-off (PTO) system and is given by:

$F_{u}(t)=-B(t) \dot{x}(t)$

\section{Parameters}

The following is a list of parameters for the hydrodynamic model described by Eq. 5 .

- $\quad m_{b}=9700 \mathrm{~kg}$

- $m_{r}(\infty)=8700 \mathrm{~kg}$

- $S=86.4 \mathrm{kN} / \mathrm{m}$

- $F_{m}=173 k N$

- $R_{f}=200 \mathrm{~kg}$

The force due to the end stop devices was left out for this study, in order to investigate unconstrained oscillation of the buoy under certain conditions.

\section{Model Simplification}

This paper investigates manipulation of the system damping force in order to achieve optimal energy recovery from a point absorber WEC. Since manipulation of the system damping during operation gives a time varying system, any mathematical manipulation on this model quickly become very cumbersome, resulting in intractable solutions and little intuitive appeal. Therefore, a simpler linear differential equivalent (still with a varying damping parameter) is determined, which will form the basis for the analytical manipulations presented in this paper, with the results validated on the original model presented in Eq. 5.

Essentially, Eidsmoen's hydrodynamic model (Eq. 5) is in the form of a simple mass, spring, damper model, with various expressions for the coefficients of displacement, velocity and acceleration. The approach here is to simplify Eidsmoen's model by approximating its parameters by the three parameters, $M, B$ and $K$, of the simple model given in Eq. 7.

$M \ddot{x}(t)+B \dot{x}(t)+K x(t)=F(t)$

Eidsmoen's hydrodynamic model parameters are approximated by the parameters of the simple model (Eq. 7) as shown in Table 1.

Table 1. Approximating the hydrodynamic model

\begin{tabular}{|l|l|l|}
\hline \multicolumn{1}{|c|}{ Coeff. Of } & Simple Model & \multicolumn{1}{c|}{$\begin{array}{c}\text { Eidsmoen's Hydrodynamic } \\
\text { Model }\end{array}$} \\
\hline Acceleration & $M$ & $m_{b}+m_{r}(\infty)$ \\
\hline Velocity & $B$ & $R_{f}+B+$ radiation convolution \\
\hline Displacement & $K$ & $S$ \\
\hline & & \\
\hline Excitation & $A$ & Excitation convolution \\
\hline & $\omega_{w}$ & $\omega_{w}$ \\
\hline & & \\
\hline others & - & $F_{m}$ \\
\hline
\end{tabular}

For the purposes of this paper the incident waves are assumed to be 
monochromatic, with the excitation force, as given by Eq. 2 in the hydrodynamic model, being approximated as:

$$
F(t)=A \sin \left(\omega_{w} t\right)
$$

In achieving the simplification of the hydrodynamic model, both the radiation and excitation kernels of the convolution integrals in Eq. 5 can be approximated as constants. This can be achieved in a number of ways. In Eidsmoen (1995), plots of both kernels are given. One possibility is to determine a relatively simple expression for these kernels, so that they can be transformed to the frequency domain (using the Laplace transform) and a dc equivalent found by setting $s=0$. Otherwise, if the expressions for the kernels do not permit this, finding the areas under the kernels will give a comparable result. Determination of the area under the kernel functions yields:

- Radiation kernel constant equivalent: 722.1 , and

- Excitation kernel constant equivalent: 43195.

In the approximation of Eidsmoen's hydrodynamic model parameters, the approximation of $A$ is given by multiplying the wave amplitude by the approximation of the excitation kernel.

Finally, since $F_{m}$, the net buoyancy force, is only a constant positive offset in the equations of motion (Eq. 5), it can be neglected in the simplified model. This is because the issues this paper investigate, such as the shape of the optimal damping profile, will not be affected by an offset.

The accuracy of the simplified model is judged throughout the paper, with the results developed using the simplified model, being validated against Eidsmoen's original hydrodynamic model.

\section{Power and Energy}

In a wave energy device, the power is developed in the damper (Ringwood and Butler, 2004), which normally represents the PTO system, as:

$$
P_{d}=\text { force } \times \text { velocity }=B \dot{x} \dot{x}=B \dot{x}^{2}
$$

The energy developed in the damper over a period of time $t_{l}$ is:

$$
E_{d}\left(t_{1}\right)=\int_{0}^{t_{1}} P_{d} d t=\int_{0}^{t_{1}} B \dot{x}^{2} d t
$$

Maximum energy is transferred to the damper when Eq. 10 is maximized over a period of the wave force. For the model of Eq. 7, this results in the condition:

$\omega_{n}=\sqrt{\frac{K}{M}}=\omega_{w}$

where $\omega_{n}$ is the natural frequency of the device and $\omega_{w}$ is the incident wave frequency. Under this maximum condition, the velocity profile of the device is in phase with the wave force, consistent with condition one in the introduction.

\section{OPTIMAL DAMPING PROFILE}

As noted in the introduction, optimal energy recovery from a point absorber WEC can be achieved if the velocity profile of the device is in phase with the excitation force experienced by the device. A huge range of possibilities exist for the parameterization of the damping term in order to achieve this. Fig. 2 illustrates the damping profiles of two such solutions known as latching and freewheeling.

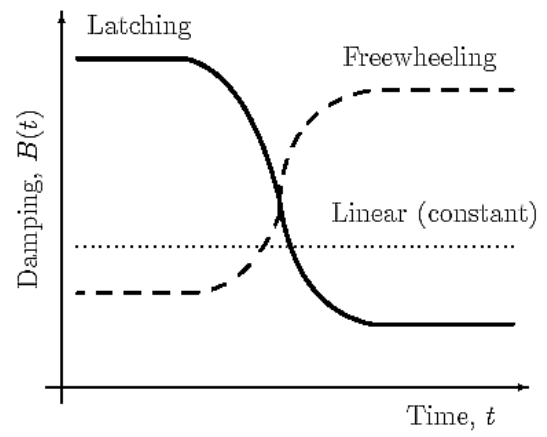

Figure 2. Possible damping profiles

It can be seen that the profiles of both solutions are in stark contrast to each other. Latching initially employs a large damping value, effectively locking the device in position, whereas freewheeling initially employs a tiny damping value in order to achieve velocity build up. Since the wave energy absorbed is converted in the damping term (see Eq. 10), the question arises as to which of these profiles is optimal in terms of energy conversion, or is it one of the multitude of other profile possibilities, that is optimal?

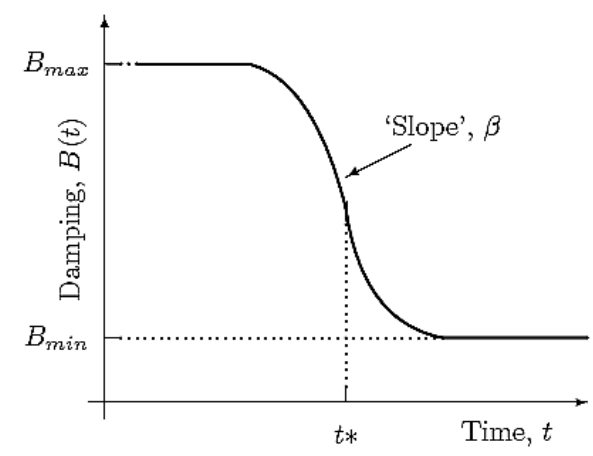

Figure 3. Sigmoidal parameterisation of damping functions

To determine the optimal loading regime, the damping term was parameterized as a general sigmoid function (see Fig. 3) as follows:

$B(t)=\frac{B_{\max }-B_{\min }}{1+e^{-\beta(t-t *)}}+B_{\min }$

This provides for many possible damping profiles, including those illustrated in Fig. 2.

The parameters of the sigmoid were adapted, using a genetic algorithm (Goldberg, 1989), in order to maximize the energy function (Eq.10) over a wave period, where $\omega_{w}=0.5 \mathrm{~Hz}$ (giving a wave period $\approx 12.5$ seconds). Since the objective is to determine the optimal damping profile for a generic point absorber, and the optimal damping profile shape is assumed to be independent of the specific device parameters, the simplified model (Eq. 7) with default values of $M=K=1$ were used. A genetic algorithm (GA), with elitism, was employed since the performance surface to be searched is non-convex with respect to the sigmoid parameters. Briefly, the parameters of the GA are given in 
Table 2, along with the allowable ranges for the sigmoid parameters in Table 3. The final values attained following maximization of the energy captured per wave period are given in Table 4.

Table 2. GA parameters

\begin{tabular}{|l|l|}
\hline Parameter & Value \\
\hline Chromosone coding & Binary \\
Population size & 70 \\
Number of generations & 30 \\
Generation gap & 0.7 \\
Recombination probability & 0.7 \\
Mutation probability (per bit) & 0.035 \\
Selection & Roulette wheel \\
\hline
\end{tabular}

Table 3. Range for sigmoid parameters

\begin{tabular}{|l|l|l|l|l|}
\hline Range & $B_{\min }$ & $B_{\max }$ & $t^{*}$ & $\beta$ \\
\hline Min & 0 & 0 & -20 & -3000 \\
Max & 50 & $10^{90}$ & +20 & 0 \\
\hline
\end{tabular}

Table 4. Final sigmoid parameter values

\begin{tabular}{|l|l|l|l|l|}
\hline Parameter & $B_{\min }$ & $B_{\max }$ & $t^{*}$ & $\beta$ \\
\hline Final value & 0.0546 & $10^{90}$ & 2.7 & -2530 \\
\hline
\end{tabular}

The final values of the sigmoid parameters clearly point to a latching profile, indicating the optimality of a latching strategy. Firstly, the value of $\beta$ is negative, indicating a "high then low" strategy. The value of $B_{\max }$, the initial damping value, goes to the upper limit of its allowable range, effectively indicating infinite initial damping (latched). The magnitude of the slope $\beta$ is very large, indicating an instantaneous transition from the latched phase to a subsequent finite value of damping.

Figs. 4 and 5 illustrate the variations in the converted energy using a latching strategy and the optimal latching period (respectively), for variations in damping and wave frequency. The default values of $M=$ $K=1$ were again used. The final value of damping returned for the optimized sigmoid parameters $\left(B_{\min }=0.0546\right)$ is very close to the optimal damping value indicated in Fig. 4, for a wave frequency of $\omega_{w}$ $=0.5$. Also, the time at which the transition from $B_{\max }$ to $B_{\min }$ takes place $\left(t^{*}=2.7 \mathrm{secs}\right)$ is very close to the optimal latching time $(\approx 2.75$, for $\omega_{w}=0.5$ ) indicated in Fig. 5.

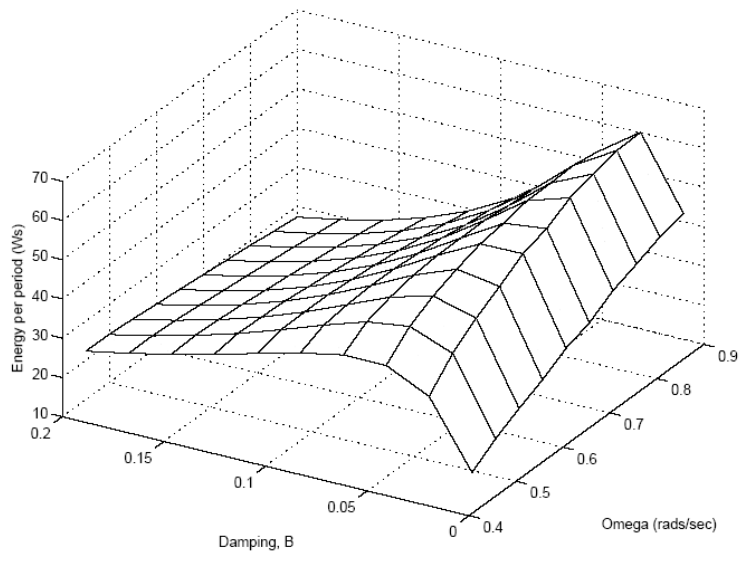

Figure 4. Variations in $E_{d}$ with $B$ and $\omega_{w}$

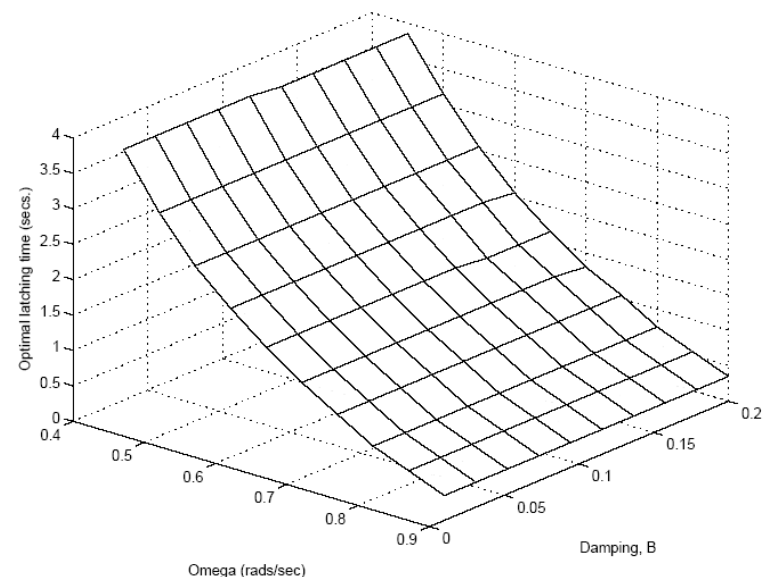

Figure 5. Variations in $T_{L}{ }^{\text {opt }}$ with $B$ and $\omega_{w}$

\section{Implementing latching in the hydrodynamic model simulation}

In simulation of Eidsmoen's hydrodynamic model (Eq. 5), latching can be achieved in a more efficient way then setting $B$ to $\infty$ (requires very fine step-size and hence large simulation times), as follows:

- The latching point is determined as the point where the device velocity goes to zero. At this point, the first and second derivatives of displacement go to zero in Eq. 5 and if the wave force is replaced by a force equal and opposite to all the non zero forces, latching is achieved. These forces are $F_{m}$, $S x(t)$ and the convolution integral term of $F_{r}(t)$. The last of these forces is included, as it consists of a convolution of previous velocity points and not just the current velocity equal to zero. The complete simulation configuration can be seen in Fig. 6.

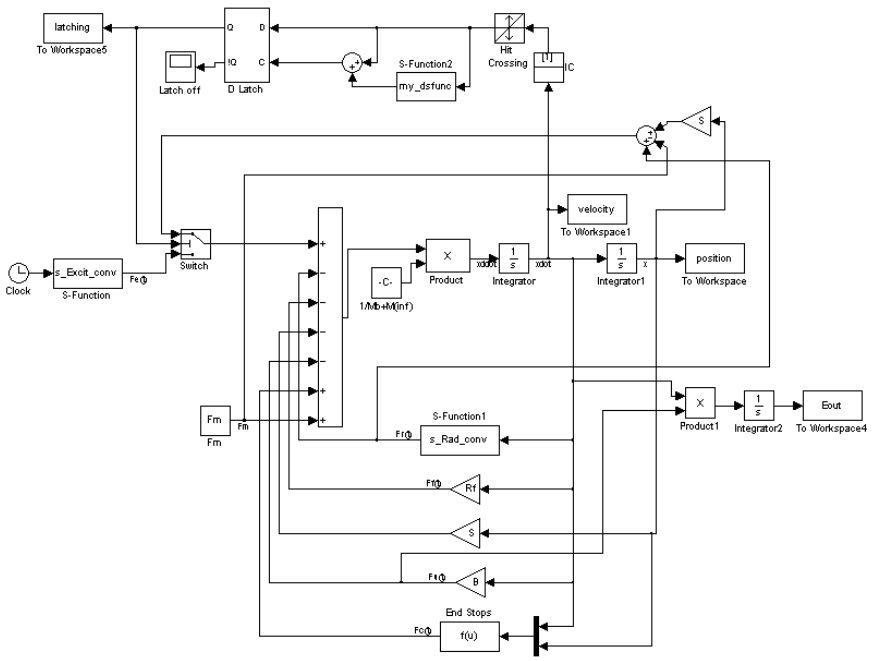

Figure 6. Simulation configuration

\section{LATCHING DETAILS}

As previously noted, a latching strategy consists of holding the device in position until the ideal moment of release, thus delaying the device velocity profile so that it becomes in phase with the wave excitation force. An illustration of this, for the simplified model (Eq. 7) with 
default values of $M=K=1$, is in Fig. 7. Latching can be achieved by means of a mechanical brake or open/close valves on the hydraulic lines of the PTO system. The instant of latching is imposed by the dynamics of the device itself and is the instant at which the velocity of the buoy dies to zero. After the holding/latching period, the device is then released to a finite damping value chosen by weighing up energy absorption against design limitations on the amplitude of the oscillation of the buoy. From Fig. 5, it can be seen that the choice of damping value has insignificant effect on the optimum latched time period. Thus, the single and most crucial control variable, for a point absorber system employing a latching strategy, becomes the duration of the latched phase.

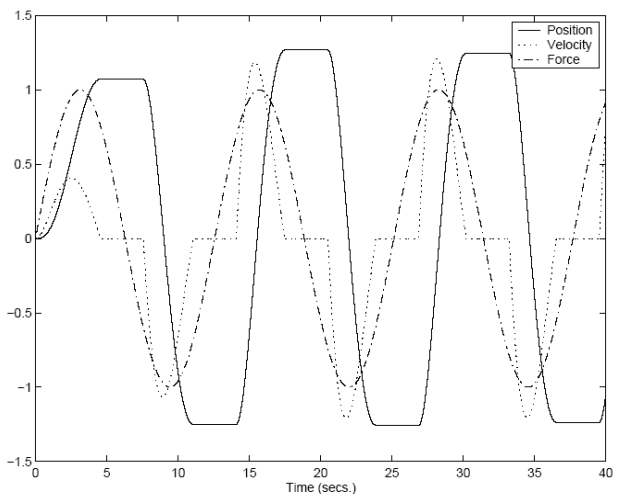

Figure 7. Latching simulation plot

\section{SEMI-ANALYTICAL SOLUTION FOR OPTIMAL LATCHING PERIOD}

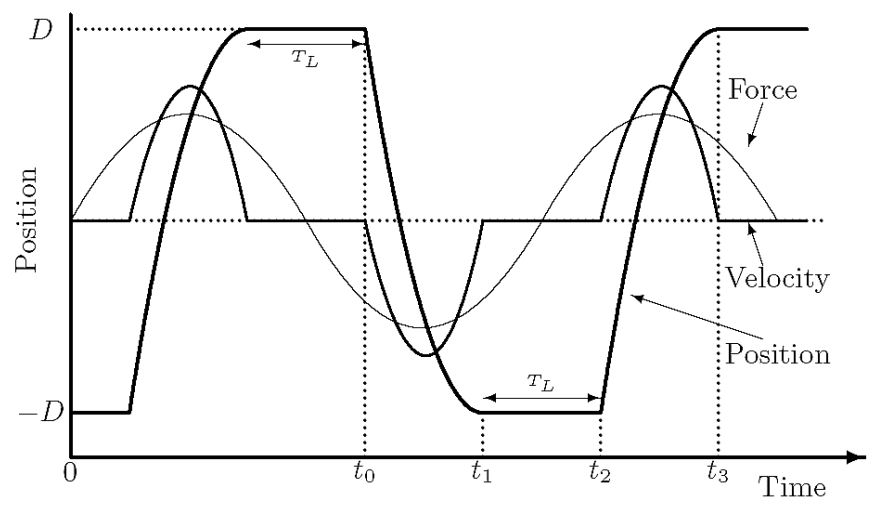

Figure 8. Latching calculations

A solution to the latched system can be attempted by considering Fig. 8. One period of the stimulus and response is given by two latched periods and two periods of free motion. During the latched period, the buoy is held in position and has zero velocity. During the periods of motion, the motion is governed by the equation of motion (Eq. 7) of the system. Given that each latching period occurs consistently for $T_{L}$ seconds, this gives the dynamic response period as:

$t_{1}-t_{0}=t_{3}-t_{2}=T_{w} / 2-T_{L}$

Assuming the transient response has died down, the solutions over $t_{0} \rightarrow$ $t_{1}$ and $t_{2} \rightarrow t_{3}$ are equal and opposite. In each case, the body will move from a position $D$ to $-D$ or vice versa.

The explicit solution for the period $t_{0} \rightarrow t_{1}$ is given by:

$$
\begin{aligned}
& x=\alpha e^{-(B / 2 M) t} \sin \left(\omega_{d} t+\delta\right)+\beta \sin \left(\omega_{w} t-\phi\right) \\
& \text { and } \\
& \dot{x}=\alpha \sqrt{K / M} e^{-(B / 2 M) t} \cos \left(\omega_{d} t+\psi+\delta\right)+\beta \omega_{w} \cos \left(\omega_{w} t-\phi\right)
\end{aligned}
$$

with,

$$
\begin{aligned}
& \omega_{d}=\sqrt{(K / M)-\left(B^{2} / 4 M^{2}\right)} \\
& \phi=\tan ^{-1}\left(\left(B \omega_{w}\right) /\left(K-M \omega_{w}^{2}\right)\right) \\
& \beta=A / \sqrt{\left(K-M \omega_{w}^{2}\right)^{2}+\left(B \omega_{w}\right)^{2}} \\
& \gamma=\sqrt{1-\left(B^{2} / 4 K M\right)} \quad ; \quad \psi=\cos ^{-1} \gamma
\end{aligned}
$$

The initial conditions for the motion starting at $t_{0}$ are:

$x=D, \dot{x}=0$

and the final conditions at $t_{1}$ are:

$x=-D, \dot{x}=0$

Using Eqs. 14 and 15 with the boundary conditions (Eqs. 20 and 21) results in the following system of equations:

$$
\begin{aligned}
& \alpha e^{-(B / 2 M) t_{0}} \sin \left(\omega_{d} t_{0}+\delta\right)+\beta \sin \left(\omega_{w} t_{0}-\phi\right)=D \\
& \alpha \sqrt{K / M} e^{-(B / 2 M) t_{0}} \cos \left(\omega_{d} t_{0}+\psi+\delta\right)+\beta \omega_{w} \cos \left(\omega_{w} t_{0}-\phi\right)=0 \\
& \text { and } \\
& \alpha e^{-(B / 2 M) t_{1}} \sin \left(\omega_{d} t_{1}+\delta\right)+\beta \sin \left(\omega_{w} t_{1}-\phi\right)=-D \\
& \alpha \sqrt{K / M} e^{-(B / 2 M) t_{1}} \cos \left(\omega_{d} t_{1}+\psi+\delta\right)+\beta \omega_{w} \cos \left(\omega_{w} t_{1}-\phi\right)=0
\end{aligned}
$$

Recombination and cancellation, in order to remove the two unknowns, $\alpha$ and $\delta$, gives:

$$
\begin{gathered}
{\left[\begin{array}{cc}
\cos \left(\omega_{d}\left(t_{1}-t_{0}\right)-\psi\right) & -\gamma e^{(B / 2 M)\left(t_{1}-t_{0}\right)} \\
\gamma e^{-(B / 2 M)\left(t_{1}-t_{0}\right)} & -\cos \left(\omega_{d}\left(t_{1}-t_{0}\right)+\psi\right)
\end{array}\right]\left[\begin{array}{c}
D-\beta \sin \left(\omega_{w} t_{0}-\phi\right) \\
-D-\beta \sin \left(\omega_{w} t_{1}-\phi\right)
\end{array}\right]} \\
=\sqrt{M / K} \sin \left(\omega_{d}\left(t_{1}-t_{0}\right)\right)\left[\begin{array}{c}
\beta \omega_{w} \cos \left(\omega_{w} t_{0}-\phi\right) \\
\beta \omega_{w} \cos \left(\omega_{w} t_{1}-\phi\right)
\end{array}\right]
\end{gathered}
$$

With some multiplication and rearrangement, the system of equations in Eq.26, result in two equations for $D$. While there are some small differences between these two equations, in terms of a number of signs and arguments of the trigonometric terms, both equations give the same result for $D$. Taking the result from manipulation of the top line of the matrices in Eq. 26 gives:

$$
\begin{aligned}
D & =\beta\left[\omega_{w} \sqrt{M / K} \sin \left(\omega_{d}\left(t_{1}-t_{0}\right)\right) \cos \left(\omega_{w} t_{0}-\phi\right)+\right. \\
& \left.\cos \left(\omega_{d}\left(t_{1}-t_{0}\right)-\psi\right) \sin \left(\omega_{w} t_{0}-\phi\right)-\gamma e^{(B / 2 M)\left(t_{1}-t_{0}\right)} \sin \left(\omega_{w} t_{1}-\phi\right)\right] \\
& /\left[\cos \left(\omega_{d}\left(t_{1}-t_{0}\right)-\psi\right)+\gamma e^{(B / 2 M)\left(t_{1}-t_{0}\right)}\right]
\end{aligned}
$$

This gives an expression for $D$ in terms of the system parameters, the excitation parameters and the times, $t_{0}$ and $t_{1}$. However, through investigation of Fig. 8 and some simple relationships, the equation for $D$ can be rewritten so it is solely a function of the system and excitation parameters, as: 


$$
\begin{aligned}
D & =\beta\left[\omega_{w} \sqrt{M / K} \sin \left(\omega_{d}\left(T_{w} / 2-T_{L}\right)\right) \cos \left(\omega_{w}\left(T_{w} / 2+T_{L} / 2\right)-\phi\right)\right. \\
& +\cos \left(\omega_{d}\left(T_{w} / 2-T_{L}\right)-\psi\right) \sin \left(\omega_{w}\left(T_{w} / 2+T_{L} / 2\right)-\phi\right) \\
& \left.-\gamma e^{(B / 2 M)\left(T_{w} / 2-T_{L}\right)} \sin \left(\omega_{w}\left(T_{w}-T_{L} / 2\right)-\phi\right)\right] \\
& /\left[\cos \left(\omega_{d}\left(T_{w} / 2-T_{L}\right)-\psi\right)+\gamma e^{(B / 2 M)\left(T_{w} / 2-T_{L}\right)}\right]
\end{aligned}
$$

since, in the optimal case, the velocity profile of the buoy is in phase with the wave excitation force, the time point, $t_{0}$, will be equal to half the wave period plus half the latched time period:

$$
t_{0}=T_{w} / 2+T_{L} / 2
$$

and an expression for $t_{1}$ can be derived through addition of Eq. 13 and Eq. 29:

$$
\begin{aligned}
t_{1} & =\left(t_{1}-t_{0}\right)+t_{0} \\
& =T_{w} / 2-T_{L}+T_{w} / 2+T_{L} / 2 \\
& =T_{w}-T_{L} / 2
\end{aligned}
$$

$D$, as expressed in Eq. 28, is now a function only of the system parameters, the wave excitation parameters and the latching time period, $T_{L}$.

From our experimental observations, there appears to be a consistency between maximizing $D$ and maximizing $E_{d}$ (also confirmed by the work of Babarit et al (2003)), allowing a complete analytical solution for the optimal latching period to be derived by setting:

$$
d D / d T_{L}=0
$$

in Eq. 28 and solving for $T_{L}$. However, the equation becomes intractable due to the presence of the $T_{L}$ parameter in a number of the trigonometric and exponential terms of the equation.

However, as illustrated in Fig. 9, the variation in $D$ with $T_{L}$, using Eq. 28 , is very smooth with a clear maximum. This allows the equation to be quickly and easily optimized in order to return the optimal latching period. Optimization, using a simple quasi-Newton constrained minimization routine (Han, 1977) (using the Matlab ${ }^{\text {TM }}$ implementation "fmincon" (Mathsworks, 2004)), returned, in this case, the optimal value of $T_{L}$ as 3.15 seconds, which matches exactly the optimal latching period, $T_{L}^{o p t}$, given in Fig. 5 (again $\omega_{w}=0.5, M=K=1$ and $B=0.2$ ).

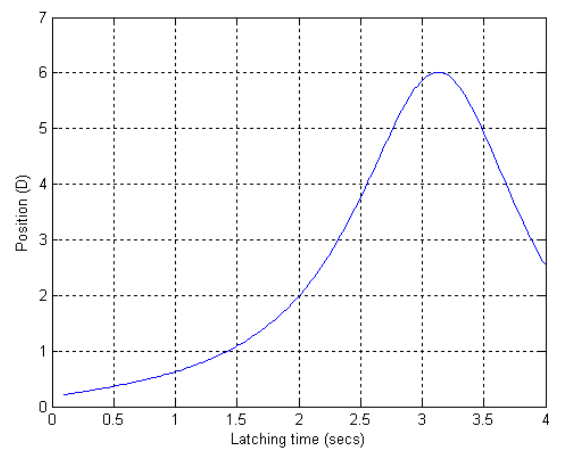

Figure 9. Variations in $D$ with $T_{L}$

In summary, a solution to find $T_{L}^{\text {opt }}$, the latching period which gives the maximum possible energy capture over a single wave period has been developed. It entails optimizing Eq. 28 for the current system and excitation parameters.

\section{Accuracy of Solution for Eidsmoen's Hydrodynamic Model}

As noted in the previous section, the solution to find the optimum latching period, $T_{L}^{\text {opt }}$, for the default values of $M=K=l$ and the wave frequency, $\omega_{w}=0.5$, gives exactly the same results as obtained from simulation of Eq. 7 for the same parameter values. In fact, the solution shows exact correlation with the simulation results of Eq. 7, for all valid values of $M, B, K, A$ and $\omega_{w}$. The real question is: how accurate is the solution for $T_{L}{ }^{\text {opt }}$, using the simplified hydrodynamic model parameters, to the simulation results of the original hydrodynamic model developed by Eidsmoen? The answer is, very accurate. This is not surprising as only the parameters $m_{b}, m_{r}(\infty), S$ and $\omega_{w}$, all of which are constants in the hydrodynamic model, are crucial to the calculation of $T_{L}^{\text {opt }}$, since:

- $\quad F_{m}$, the net buoyancy force, can be neglected, as it is only a constant positive offset in the equations of motion and hence won't effect $T_{L}{ }^{\text {opt }}$,

- As previously discussed, the damping term, $B$, has an insignificant effect on $T_{L}^{\text {opt }}$ (see Fig. 5, variation in $B$ has little effect on $T_{L}^{o p t}$ ) and hence its approximation does not need to be too accurate, and

- As illustrated in Fig. 10, changing $A$, the amplitude of the excitation force, also has an insignificant effect on $T_{L}{ }^{o p t}$. The figure shows the variation of $D$ with $T_{L}$ for three different excitation amplitudes and in each case $T_{L}^{\text {opt }}$ is the same. This means that the approximation of $A$ doesn't need to be very accurate either.

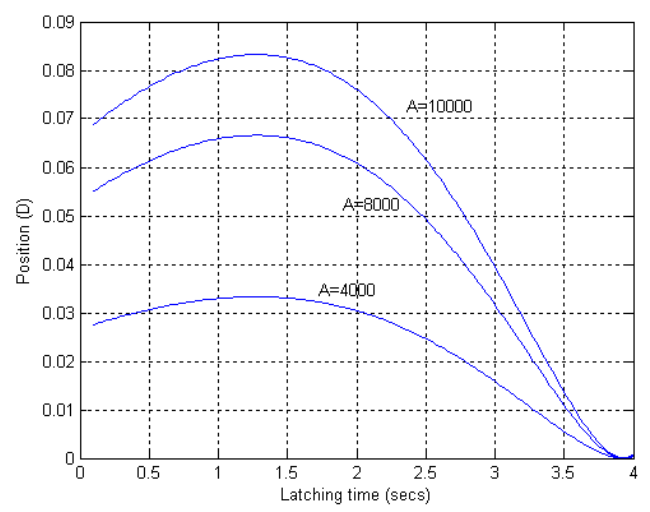

Figure 10. Variations in $D$ with $T_{L}$, for different force amplitudes, $A$

The results from approximating Eidsmoen's hydrodynamic model parameters as single values of $M, B, K, A$ and $\omega_{w}$ and using the solution developed to determine $T_{L}{ }^{\text {opt }}$, are very accurate. Fig. 11 shows the energy capture per period, with variation in $T_{L}$ and $B=22000$, calculated through simulation of the hydrodynamic model of Eq. 5 . The clear optimal $T_{L}$, in terms of energy capture, is 2.45 seconds. This is in good correlation with Fig. 12, which illustrates the variation in $D$ with $T_{L}$ (using Eq. 28 and the approximated hydrodynamic parameters), again showing the optimal $T_{L}$, in terms of maximization of $D$, to be 2.45 seconds. 


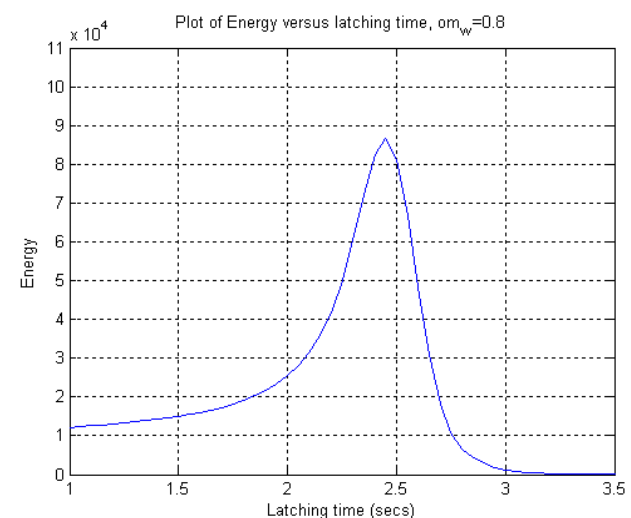

Figure 11. Variations in energy capture with $T_{L}$

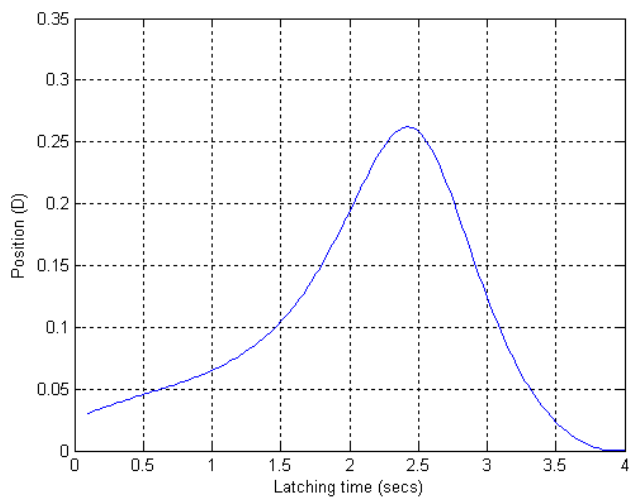

Figure 12. Variations in $D$ with $T_{L}$

In summary, this section has detailed a quick and efficient (could be used on a wave by wave basis) solution, for finding the optimal latching period, in terms of energy capture, for the complete hydrodynamic model (Eq. 5) of a point absorber WEC, as developed by Eidsmoen (1995). It is possible that this solution could be used in conjunction with a real point absorber WEC operating at sea.

\section{CONTROL CONSIDERATIONS}

It is envisaged that some short term forecasting, to predict the incident waves, may be needed in order to implement a latching strategy in the real world. The controller would work as follows:

- Given the system parameters and the current wave excitation parameters, solve for $T_{L}^{\text {opt }}$ by optimizing Eq. 28,

- Calculate $D^{\text {opt }}$, the amplitude of oscillation for $T_{L}^{\text {opt }}$, by substituting $T_{L}{ }^{\text {opt }}$ back into Eq. 28,

- If $D^{\text {opt }}<D^{\max }$, the designed amplitude oscillation limit, use $T_{L}^{\text {opt }}$,

- Else, if $D^{o p t}>D^{\max }$ solve Eq. 28 to give $T_{L}^{\text {best }}$, the latching period giving $D=D^{\max }$, and use this as the latching period.

It has already been shown that the solution developed to determine $T_{L}{ }^{\text {opt }}$, for Eidsmoen's hydrodynamic model, is very accurate. It has also been noted that only the parameters $m_{b}, m_{r}(\infty), S$ and $\omega_{w}$, all of which are constants in the hydrodynamic model, are crucial to the accuracy of $T_{L}^{\text {opt }}$. However, if a control algorithm like the one above is to be used, then all the parameters are needed to enable accurate calculation of $D$. This means that approximation of the hydrodynamic model parameters, as the parameters of the simplified model, as detailed in Table 1, need to be as accurate as possible.
It turns out, that the calculation of $D$ using the approximated hydrodynamic parameters, does not give accurate correlation to the simulation results of Eidsmoen's hydrodynamic model (Eq. 5). This is because, the constant equivalents used as approximations of the radiation and excitation kernels of Eq. 5, are not very accurate approximations. For example, the approximation of $A$, for a wave amplitude of one meter, using the constant equivalent, is 43195. However, in simulation of the hydrodynamic model, the amplitude of the wave excitation force as the result of the convolution integral of $F_{e}(t)$, for the same wave amplitude, is 31680 . This discrepancy is noticeable in the calculation of $D$, with the approximation of $A$ as 43195 giving a value of $D, 0.5$ meters greater then that of the simulation. If the value of $A$ is changed to 31680 the difference is reduced to 0.1 meters. However, in the real world, it is envisaged that the excitation parameters will be supplied by some form of wave forecasting or possibly calculated from information supplied by a wave rider in front of the device. This will allow a more accurate approximation of the amplitude of the excitation force, $A$, and will allow the use of Eq. 28 to give an accurate calculation of $D$.

\section{Damping Effect}

Since there are other demands on one of the system parameters, the control algorithm, given across, becomes constrained. As illustrated in Fig. 5, $T_{L}^{\text {opt }}$ is largely independent of the damping term $B$. However, $B$ does affect both the amount of energy captured and the amplitude of oscillation of the buoy.

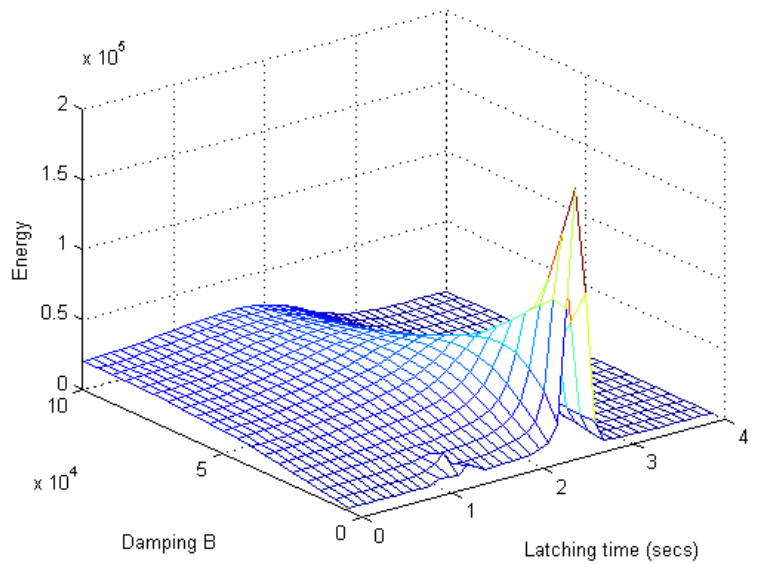

Figure 13. Variations in energy captured with $B$ and $T_{L}$

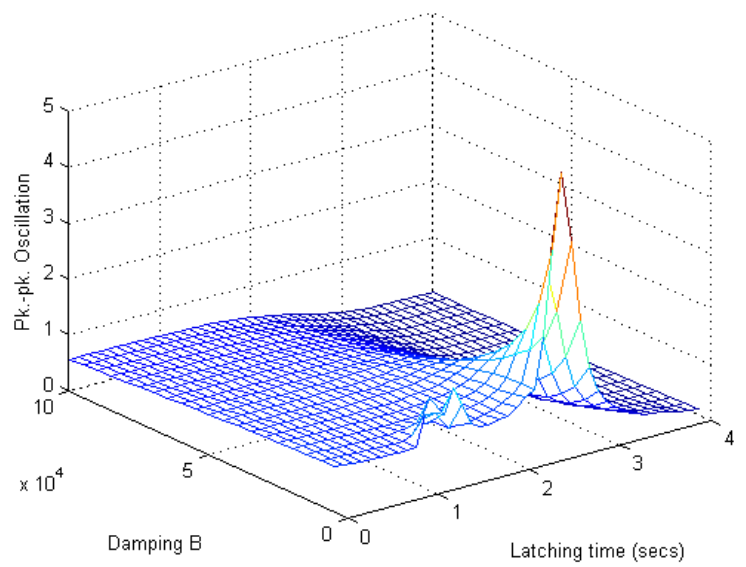

Figure 14. Variations in peak to peak oscillation with $B$ and $T_{L}$ 
Fig. 13 illustrates, for Eidsmoen's hydrodynamic model, the energy captured across a single wave period with variations in damping and latching period. There is a clear optimal combination of $B$ and $T_{L}$, which captures significantly more energy, then the optimal $B$ when there is no latching $\left(T_{L}=0\right.$, optimal $\left.B=100,000\right)$. However, at this optimal combination the amplitude of oscillation of the buoy becomes extremely large (see Fig. 14). Here, the validity of the model becomes questionable with the degree of peakness possibly being a characteristic of the model itself. Nonetheless, the large oscillations of the buoy are undesirable. Another noteworthy point comes from investigation of Fig. 15. It illustrates the variation in $D$ with $T_{L}$ for two different damping values $(B=1000$ and 15000$)$. It can be seen that $T_{L}^{o p t}$ is the same for both damping values. However, $D$ is much more sensitive to $T_{L}$ for the smaller damping value. This means that when operating at small values of $B$, any inaccuracy in the calculation or implementation of the latching period will be severely punished with a huge shortfall in the expected energy capture.

Both the extremely large oscillations of the buoy and the high sensitivity of $D$ to $T_{L}$, occur at relatively small values of damping ( $B<$ 5000). However, it is in this region that energy capture is at its highest. It is due to this confliction of interest with $B$, that the calculation and optimization of the optimum latching period becomes constrained, even though it was previously established that the choice of $B$ does not directly effect the calculation of the optimal latching period.

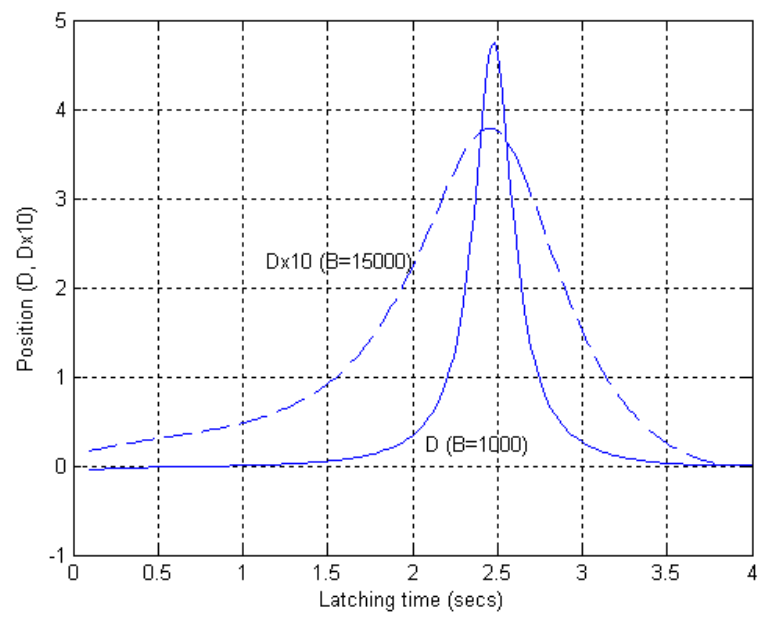

Figure 15. Variation in $D$ with $T_{L}$ at $B=1000$ and 15000

\section{CONCLUSIONS}

Optimal extraction of wave energy requires a number of device aspects to be considered. Firstly, the system must be designed so that the resonant frequency of the device is equal to the predominant wave frequency at the device deployment location. Further to this, provision must be made for when the wave frequency deviates away from the predominant wave frequency. This paper has established that latching is the optimal strategy, in terms of energy conversion, to bring the device velocity profile back in phase with the wave excitation force, thus meeting condition one, given in the introduction, for optimal energy recovery.

A solution for the single most crucial control variable when adopting a latching strategy, the latching period, has been developed and validated for a detailed hydrodynamic model of a point absorber WEC. It has been clarified, that further care needs to be taken in setting the appropriate (unlatched) damping value. Further work needs to be carried out in assessing if the gain in energy extraction, when employing a latching strategy, is significant enough to offset the added costs in expenditure, reliability and complexity of employing a latching strategy.

\section{ACKNOWLEDGEMENTS}

We would like to thank the referees for their useful comments and we would like to acknowledge the financial support provided by the Postgraduate Travel Fund at NUI Maynooth.

\section{REFERENCES}

Babarit, A, Duclos, G, and Clement, AH (2003). "Comparison of Latching Control Strategies for a Heaving Wave Energy Device in Random Seas," Proc. $5^{\text {th }}$ European Wave Energy Conference, Cork, Ireland.

Budal, K, and Falnes, J (1975). "A Resonant Point Absorber of Ocean Wave Power," Nature, Vol 256, pp 478-479.

Cummins, WE (1962). "The Impulse Response Function and Ship Motions," Schiffstechnik, Vol 9, pp 101-109.

Eidsmoen, H (1995). "On Theory and Simulation of Heaving-Buoy Wave-Energy Converters with Control," PhD. Thesis, Division of Physics, Norwegian Institute of Technology, Trondheim, Norway.

Falnes, J (2002). "Ocean Waves and Oscillating Systems," Cambridge University Press, Cambridge, UK.

Goldberg, DE (1989). "Genetic Algorithms in Search, Optimization and Machine Learning," Addison-Wesley.

Han, SP (1977). "A Globally Convergent Method for Nonlinear Programming," Journal of Optimization Theory and Applications, Vol 22, p. 297.

Mathsworks (Accessed 2004). "Optimization Toolbox: fmincon [online]" Available from: http://www.mathworks.com/access/helpdesk/help /toolbox/optim/fmincon.html

Ringwood, JV, and Butler, S (2004). "Optimisation of a Wave Energy Converter," Proc. IFAC Conf. On Control Applications in Marine Systems, Ancona, Italy.

Wright, A, Beattie, WC, and Thompson, A (2003). "Performance Considerations in a Power Take-Off Unit Based on a Non-Linear Load," Proc. $5^{\text {th }}$ European Wave Energy Conference, Cork, Ireland. 